\title{
WEAK DISCERNIBILITY AND RELATIONS BETWEEN QUANTA
}

\author{
JOSHUA NORTON
}

\begin{abstract}
Some authors (Muller and Saunders 2008, Huggett and Norton, 2013) have attempted to defend Leibniz's identity of indicernibes through weak discernibility. The idea is that if there is a symmetric, non-reflexive physical relation which holds between two particles, then those particles cannot be identical. In this paper I focus only on Muller and Saunders' account and argue that the means by which they achieve weak discernibility is not through a physical observable but an alternate mathematical construction which is both unorthodox and incomplete. Muller and Saunders build a map from numbers to a set of observables (mostly) and out of this map construct a weakly discerning formal relation. What Muller and Saunders' do not provide is a worked out account of how such maps pick out physical relations between particles.
\end{abstract}




\section{Introduction}

In their paper, "Discerning Fermions," Muller and Saunders (2008) argue that identical particles are weakly discerned by having opposite spin. This is in response to the long-standing concern that identical particles, like fermions, violate Leibniz's Principle of the Identity of Indiscernibles (PII). However, if "identical" particles are discernible by their spins then they do not differ merely numerically and thus do not violate the PII. If Muller and Saunders are correct, then they will have successfully demonstrated that one form of the PII is immune from challenges posed by identical particles. The first half of this paper will involve laying out the relevant issues as well as Muller and Saunders' position. In the second half of this paper, I will argue that Muller and Saunders' account fails since they don't make use of quantum observables and what they do make use of cannot be interpreted as a physical relation. ${ }^{1}$ Without a physically pertinent relation, one cannot even begin the process of weak discernibility.

\section{The Challenge from Identical Particles and Weak Discernibility}

Assuming some familiarity with both the PII and with the challenge raised by identical particles,

I will be brief in my retelling of this story. Leibniz posited seven principles which he largely took as being self evident, the Principle of the Identity of Indiscernibles being one of them. This principle states that if two objects are indiscernible then they are identical or, a slightly more interesting formulation, "That it is not true that two (distinct) substances may be exactly alike and different merely numerically, solo numero," (Leibniz, 1686). I say more interesting because this quote makes

${ }^{1}$ Both Huggett and Norton (2013) as well as French and Redhead (1988), make a similar mistake in how they build physical relations. Huggett and Norton make the same assumptions as Muller and Saunders.' French and Redhead consider relations built out of conditional probabilities of single particle operators and provide no argument that these relations represent physical relations of the particles themselves. See Huggett and Norton (2013) for remarks on French and Redhead's use of non-symmetrized observables. 
it clear that if we know there are two numerically distinct things then they must be discernible; however, it also says that if two things are indiscernible then they must not be even numerically distinct-really there is only one things.

The principle can be written as:

$$
\forall x \forall y[\forall F \in\{F\} \mid(F(x) \equiv F(y)) \supset(x=y)]^{2}
$$

Where $\{\mathrm{F}\}$ represents the set of monadic properties for the objects in our domain. According to this formulation, two objects, $a$ and $b$ are strongly discerned if, and only if:

$$
\exists F \in\{F\} \mid\{[F(a) \wedge \neg F(b)] \vee[\neg F(a) \wedge F(b)]\}
$$

The literature recognizes other means of discerning objects including that used by Muller and Saunders: two objects, $a$ and $b$, are weakly discerned if, and only if, there is a physically relevant

\footnotetext{
${ }^{2}$ Some authors include both monadic and relational properties in the set $F$; however, Leibniz's view on relations suggest that we keep these cases separate. Leibniz scholars debate whether Leibniz was simply an antirealist regarding relations, in which case relations would certainly not be included in his PII, or whether relations, for Leibniz, are unique in the sense that no two objects could partake of the same relation as some other distinct pair of objects. For instance, the relation of brotherhood shared between two men would not the same as the relation of brotherhood shared between any other distinct pair of men. Consequently, if a relation is satisfied by a pair of distinct objects, the relation fails to be reflexive and as a consequence weak discernibility is easy to satisfy. Under this second interpretation it again seems unlikely that relations were originally included in the PII. For a discussion on Leibniz' views see Mugnai (1992).
} 
dyadic predicate that is symmetric and non-reflexive ${ }^{3}$ when applied to the two objects:

$$
\exists F(a, b) \mid[F(a, b) \wedge F(b, a) \wedge \neg F(a, a) \wedge \neg F(b, b)]^{4}
$$

The idea behind weak discernibility is that if two objects are identical then $F(a, b)$ is equivalent to $F(a, a)$ and the above condition fails; thus, if it does hold then $a$ and $b$ cannot be identical. Though the inclusion of weak discernibility shifts the debate away from the exact form of Leibniz's principle, the spirit of the debate remains very much the same.

Quantum mechanics challenges the truth of Leibniz's principle in that identical particles in states like the singlet state (4) are thought to be distinct particles and yet have no property to discern them. The term 'identical particles' is unfortunate given the current context. To be clear in the context of quantum mechanics, all that is entailed by two or more particles being identical is that each has the same value for their non-dynamical properties such as their mass and charge. Identical particles are not necessarily identical in a logical or ontological sense. For the sake of sticking as closely to the terms of Leibniz's project I will be assuming that we have two objects which do differ at least numerically, but there is an important sense in which entangled states may deny this and thereby trivialize the question of discernibility. ${ }^{5}$

${ }^{3}$ Quine (1976) as well as Muller and Saunders (2008), formulate the principle of weak discernibility in terms of irreflexive relations and not non-reflexive relations. However, I see no reason to require the logically stronger relation in the context of discerning identical particles.

${ }^{4}$ Muller and Saunders (2008, 528-529). The authors also note that weak discernibility can be satisfied by a relation which is reflexive and symmetric and which does not hold between all nonidentical objects. Examples of such relations are 'identical to', or perhaps 'same haecceity.' Such relations will not be of much use to us.

${ }^{5}$ Given the holistic nature of entangled states, one might question whether or not it even makes sense to speak of having two particles when the state is entangled. It is plausible that particles lose their identity through entanglement and become only a new unity. If this is correct, then the PII cannot be challenged by entanglement since there are no longer two things which differ solo numero. The basic question is, are entangled particles more like a pair of marbles in a blackbox, or like two lumps of clay which have been combined into one new lump? If entanglement represents something closer to clay being lumped together, then there are no longer two things to discern. 
The singlet state:

$$
\Psi=\frac{1}{\sqrt{2}}(\uparrow \downarrow-\downarrow \uparrow)
$$

Here the state of the entangled two particle system is expressed in terms of $\hat{z}$-spin, while we keep suppressed the particles' location. In order to be relevant for the PII, we insist that the implicit portion of the wave function be identical and that the particles follow the same path through space. This state is physically realizable as two entangled electrons in the same orbital of some atom. In such a situation, for all the properties that are specified by quantum mechanics, the particles share said properties. In other words, there is no property held by one of the particles and not by the other, yet we do not think that these particles are ontologically one-as would be required by the PII. Consequently, many have taken the existence of such particles as being counterexamples to the PII.

Though identical particles fail to be strongly discernible, Muller and Saunders claim that they are weakly discerned by the relation 'has opposite spin.' This relation is symmetric and irreflexive and yet in order to justify its application to the singlet state we need an 'opposite spin'-observable which when applied to the singlet state yields an appropriate eigenvalue. A common assumption, though far from obvious, is that there is a one-to-one mapping from physical properties to quantum mechanical hermitian operators (observables), such that if a state $\Psi$ is the $i$ th eigenvector of the observable with eigenvalue $a_{i}$ then the state is said to have the property associated with this eigenvalue. This association between properties and eigenvalues is often referred to as the "eigenvector, eigenvalue link" (EE-link) and serves to coordinate hermitian operators with properties of 
a system. In order for us to have a mathematical framework complete enough to describe quantum phenomena, we require that our mathematical machinery be able to represent every physical property that might be possessed by our system. Thus, if we want to play the game of modeling quantum mechanics at all, we must assume that the aforementioned mapping is at least injective. ${ }^{6}$

Muller and Saunders (533) use the following generalized operator to build the relation of having or failing to have opposite spin:

$$
\sum_{i, j=1}^{d} P_{i j}^{(a)} P_{i j}^{(b)}
$$

Without getting into too many of the details, the superscripts on the single particle operators $P_{i j}^{(a)}$ pick out which slot of the tensor product the operators act and the subscripts refer to vectors of a given eigen basis. For example, $P_{\uparrow \downarrow}^{(2)}=\mathbb{I} \otimes\left(P_{\uparrow}-P_{\downarrow}\right) \otimes \mathbb{I} \otimes \ldots$, where the $P_{\uparrow}$ projects onto the $\uparrow$ $\hat{z}$-spin eigen vector and the and $P_{\downarrow}$ onto the $\downarrow$. Muller and Saunders $(533,535)$ define the following two relations and claim that both are able to weakly discern the identical particles:

$$
\begin{gathered}
Z_{-2}(a, b) \text { iff } \sum_{i, j=1}^{d} P_{i j}^{(a)} P_{i j}^{(b)} \Psi=-2 \Psi \\
Z_{2}(a, b) \text { iff } \quad \sum_{i, j=1}^{d} P_{i j}^{(a)} P_{i j}^{(b)} \Psi=2 \Psi
\end{gathered}
$$

In the case considered by Muller and Saunders, the $P_{i j}^{(\cdot)}$ operators each reduce to the Pauli spin matrix $\sigma_{z}$ and we get the following refinement:

$$
Z_{-2}(a, b) \quad \text { iff } \quad 2 \sigma_{z}^{a} \sigma_{z}^{b} \Psi=-2 \Psi
$$

${ }^{6} \mathrm{I}$ am leaving open the question of surjectivity; in particular, if there are super selection rules then the mapping will not be surjective. 


$$
Z_{2}(a, b) \quad \text { iff } \quad 2 \sigma_{z}^{a} \sigma_{z}^{b} \Psi=2 \Psi
$$

The relation $Z_{-2}$ holds for $a=1, b=2$ as well as for $a=2, b=1$ and fails for all values where $a=b$. In other words $Z_{-2}$ holds iff $a \neq b$. The relation $Z_{2}$ holds under the exact opposite conditions. Thus the particles are weakly discerned by the relation $Z_{-2}$ and the PII is immune from challenges posed by identical particles. For a more detailed account see Muller and Sauders (2008).

Although Muller and Saunders' account seems unproblematic it is not. Equation (8) is really three equations each with its own observable. As we select particular values for $a$ and $b$ we construct new mathematical objects, see (10). Consequently, the formal relation $Z_{-2}$, used to weakly discern, is not associated with an observable on the system but with three. Given that Muller and Saunders associate a single property to (8), the natural question to ask is "how do the many observables work together to jointly pick out a single dyadic property? Moreover, why should a single physical relation be identified by the set of observables in (8) and not a monadic property about the system? Or, why are not three distinct properties identified by the three distinct observables? These questions are essential for the success of Muller and Saunders' program for without a dyadic property one cannot get weak discernibility off the ground. Explicating this criticism will comprise the remainder of this paper. 


\section{Challenges}

Muller and Saunders are aware that traditionally the EE-link (roughly what they refer to as $\mathrm{StrPP})^{7}$ is used to pick out monadic predicates, they none-the-less insist that it can also pick out polyadic predicates:

Finally, a note on relations. When physical system $\mathrm{S}$ is (taken as) a composite system, built up of other physical systems, some of the properties of S determine and are determined by relations of its constituents. ... Consequently, both WkPP and StrPP [EE-link], although giving rise to properties of S and of its subsystems (expressed by monadic predicates), equally provide conditions for the ascription of relations among constituents of S; the magnitude A may itself be relational (as in relative distance), and likewise the operator A corresponding to it. (This is why one does not need to introduce relation postulates in addition to property postulates.) Typically, however, as we shall see in the next section, where authors have made use of $\mathrm{WkPP}$ or StrPP [EE-link], they have used them only to consider monadic properties - that is to say, from our point of view, they have not made use of either property postulate to ascribe relations among constituents of S which is the key step that we shall be taking in this paper.

(Muller and Saunders, 515)

According to Muller and Saunders, the following are the conditions for ascribing a physical relation to a formal relation as in (8):

${ }^{7}$ According to Muller and Saunders "We represent a quantitative physical property mathematically by the ordered pair $\langle A, a\rangle$ where $A$ is the operator which corresponds to physical magnitude $\mathcal{A}$ and $a \in \mathbb{C}$ is its value. According to the Strong Property Postulate (StrPP), a physical system $S$ having state operator $W \in \mathcal{S}(\mathcal{H})$ possesses quantitative physical property $\langle A, a\rangle \in \mathcal{M S}(\mathcal{H}) \times \mathbf{C}$ iff $W$ is an eigenstate of $A$ that belongs to eigenvalue $a "$ (513). 
When the projectors under consideration belong to the spectral family of magnitudeoperator A, assumed to be physically meaningful, they are themselves physically meaningful; by the WkPP, when the system is in the state $\mathrm{W}$, so is relation Rt, which is defined in terms of them (Req1).

(Muller and Saunders, 532)

Where the condition (Req1) is "all properties and relations should be transparently defined in terms of physical states and operators that correspond to physical magnitudes, as in WkPP, in order for the properties and relations to be physically meaningful" (Muller and Saunders, 527). Here Muller and Saunders's use the WkPP which is simply a weaker version of the StrPP (Muller and Saunders, 514) and will make no difference to my argument. In summary, according to Muller and Saunders, what justifies assigning a physical relation, 'opposite spin', to $Z_{-2}$ is merely that such a relation gains physical transparency by piggy-backing off the physical transparency of the observables which show up in (8). Before examining this claim I want to make clear that I am in no way questioning whether the observables which show up in (8) have physical meaning and am only questioning how physical content is forced onto a formal relation built out of them.

Firstly, $Z_{-2}$ is not an observable itself, but is a two-valued map from the set $\{1,2\}$ to the following set of observables:

$$
\left\{2 \sigma_{z} \otimes \sigma_{z}, \quad 2\left(\sigma_{z}\right)^{2} \otimes \mathbb{I}, \quad 2 \mathbb{I} \otimes\left(\sigma_{z}\right)^{2}\right\}^{8}
$$

$Z_{-2}$ is associated with three different observables, each of which seem to be "manifestly" physical and yet each of which could bear a distinct physical meaning. What then is the relationship between this set of observables and $Z_{-2}$ whereby the map becomes associated with a single physical relation?

${ }^{8}$ As discussed by Huggett and Norton (2013), we should be cautious of these observables since they are multiples of the identity on the space of fermions and are not symmetrized. 
There are three questions: (1) why must the map represent any physical property of the system or of the particles which make up the system, (2) why is this map associated with a relational property and not a property of some different arity, and (3) even if the mapping is associated with a relational property, why must we think that this relation is symmetric and irreflexive? Surely the formal relation $Z_{-2}(a, b)$ is symmetric and irreflexive on slot indices $a$ and $b$, but why must the observables in (8) bind together to produce a physical relation which is symmetric and irreflexive on the particles? The criterion we are in search for needs to be more than that the objects mapped onto are physically meaningful, or are individually a relation of a certain kind since in general it is false that maps have the same properties as the objects in their image.

What Muller and Saunders need to supply is an EE-link for relations which identifies when nvalued functions onto sets of observables pick out m-ary properties. Such a link would unpack what it means for a relation to be "transparently defined" by selecting which formally defined relations admit of physical predication and what the relevant physical property is. However, an EE-link for relations is neither provided for by Muller and Saunders nor is it part of standard quantum mechanics.

Let us leave aside question (1) and next consider question (2) with respect to the singlet state and 'opposite spin.' Is there some sense in which we might think it correct to assign 'opposite spin' to Muller and Saunders' relation? Let us take another look at (8):

$$
Z_{-2}(a, b) \equiv 2 \sigma_{z}^{a} \sigma_{z}^{b} \Psi=-2 \Psi
$$

For $\mathrm{a}=1, \mathrm{~b}=2(a \leftrightarrow b)$ the relation holds:

$$
Z_{-2}(1,2) \equiv 2 \sigma_{z} \otimes \sigma_{z} \Psi=-2 \Psi
$$


For $\mathrm{a}=\mathrm{b}=1$ the relation does not hold:

$$
Z_{-2}(1,1) \equiv 2\left(\sigma_{z}\right)^{2} \otimes \mathbb{I} \Psi \neq-2 \Psi
$$

For $\mathrm{a}=\mathrm{b}=2$ the relation does not hold:

$$
Z_{-2}(2,2) \equiv 2 \mathbb{I} \otimes\left(\sigma_{z}\right)^{2} \Psi \neq-2 \Psi
$$

We are granting that the map $Z_{-2}$ is physically salient and would like to use the information in (11) - (13) to select a weakly discerning relation like 'opposite spin'. Given this goal, it must not be the case that equation (11) tells us that the state has total spin equal to zero while (12) and (13) tell us that the total spin fails to be either greater or less than zero, for then it would be hard to argue that mapping is a relation since the individual observables are monadic properties of the system. Similarly, the equations must not bind together to suggest that the map represents some three place relation between the particles and their environment. Somehow we need these equations to select a two place relation on particles that is also symmetric and irreflexive. That equations (11) - (13) do select such a relation is neither argued for by Muller and Saunders nor is it even clear what such an argument would look like. Without this relation, Muller and Saunders have only a physically salient map and no way to weakly discern. So far we have not found anything in the formal structure of $Z_{-2}$ acting on $\Psi$ which suggests 'opposite spin' or any other symmetric and irreflexive physical relation. Why then think that it does? With respect to their claim that $Z_{-2}(a, b)$ represents 'opposite spin,' Muller and Saunders say:

Relation $Z_{-2}$ is the one in footnote 5 of (Saunders [2003a], p. 294): 'has opposite direction of each component of spin to.'(Muller and Saunders, 535) 
In this footnote Saunders argues:

The most general anitsymmetrized 2-particle state is $\Psi=\frac{1}{\sqrt{2}}(\phi \otimes \psi-\psi \otimes \phi)$ where $\phi$ and $\psi$ are orthogonal. Analogues of operators for components of spin can be defined as $\mathbf{S}=P_{\phi}-P_{\psi}$, where $P_{\phi}, P_{\psi}$ are projections on the states $\phi, \psi$. Each of the two particles in the state $\Psi$, has opposite value of $\mathbf{S}$. But no particle can have opposite value of $\mathbf{S}$ to itself. ${ }^{9}$

(Saunders, 2003a)

What does the heavy lifting in predicating opposite $\mathbf{S}$-value (opposite spin) to the particles is apparently Saunders' assertion that each of the two particles in the state $\Psi$ happen to have opposite values of spin. This is surely troubling; if we are not going to reason in a circle we must have some way of verifying that the particles do in fact give opposite values of $\mathbf{S}$. Yet given that the state is entangled we have no way of isolating any single particle to determine its spin. Saunders claims that the observable $\mathbf{S}$ does pick out opposite eigenvalues when applied to the particles in the singlet state; however, in order to make this claim one would need to first identify which part of the singlet state, represents some single particle. Though we know that such talk is nonsense, perhaps if we were to look at the actual terms of the $\hat{z}$-spin-expansion of the singlet state, we might be able to find some motivation for thinking that 'opposite spin' has something to do with the singlet state.

\footnotetext{
${ }^{9}$ There are formal problems with Saunders' $\mathbf{S}$ : it is a single particle observable and cannot be applied to the singlet state. This is corrected in Muller and Saunders; though, their observable is not symmetrized. In this same footnote, presumably in defense of his position, Saunders quotes Mermin (1998). In this paper Mermin gives a non-standard interpretation of quantum mechanics under which all there is, are relations and no relata. According to this interpretation there are no particles to weakly discern since there are no particles. The role which Mermin's interpretation is supposed to play in Saunders' account is far from clear. Surely such an interpretation cannot help decide the fate of the PII which assumes that there is relata in the world.
} 
It sure seems like the particles in (14) have opposite spin.

$$
\Psi=\frac{1}{\sqrt{2}}(\uparrow \downarrow-\downarrow \uparrow)
$$

How does one dissect this state to apprehend one particle at a time and thus come to believe that each particle has the opposite S-value from the other? If one were to assume that the slots of each term of the singlet state represent the individual particles, then we might conclude that the particles have opposite spin. For instance, let:

$$
\text { Particle } 1 \quad(\uparrow \otimes \cdot) \quad \text { as well as }(\downarrow \otimes \cdot)
$$

Then according to our assumption, the first slot in $\uparrow \downarrow$ represents the first particle and its properties and the second the second particle and its properties, and similarly for $\downarrow \uparrow$. Since the first particle in $\uparrow \downarrow$ has the opposite value of spin as the second particle, we conclude that the particles have opposite spin. Not forgetting that there are two terms, we check the second term $\downarrow \uparrow$ and note that the particles represented here also have opposite spin. Therefore by the linearity of (14), it is true of the singlet state that the particles have opposite spin.

The mistake of course is that $\uparrow \downarrow$ does not represent anything physical: no identical particles are represented by $\uparrow \downarrow$. The eigenvector $\uparrow \downarrow$ is no more essential to the physical description of the state than the minus sign connecting the other nonphysical pieces of syntax. As a whole, the state has physical content but none of its parts do. To treat the terms $\uparrow \downarrow$ or $\downarrow \uparrow$ as individually giving us physical information of the state is to treat the singlet state as akin to a statistical mixture rather 
than a genuine entanglement. ${ }^{10}$ This line of reasoning corrects an assumption we may have had regarding the EE-link: we may have assumed that since observables are linear in the wave function that the properties associated with them are too. This of course is false. Physical properties only hold on physical states. The EE-link associates properties with physical states and not with non-physical terms of a state's decomposition.

In summary of these arguments: the singlet state is ' $\Psi$ ' and focussing too much on the expansion '( $\downarrow-\downarrow \uparrow)^{\prime}$ may tempt us into thinking that its pattern of arrows is revealing hidden relations between the single particles. We must conclude that there is nothing in the singlet state's description that warrants attributing opposite spin to its particles. Moreover, since, as we have seen, there is nothing in the formal structure of $Z_{-2}$ which warrants our association of it to the relation 'opposite spin,' quantum mechanics simply does not provide us with a description of the particles' spins. ${ }^{11}$ If quantum mechanics does not provide a description then why do we think there is one?

I have argued that there is nothing justifying the ascription of 'opposite spin' to the singlet state; however, just because we do not have have good reasons for something does not mean that it is false. To overcome this lacuna I will provide some reason for thinking that it is in general wrong to associate properties like 'opposite spin' to entangled states. As we are aware, more information is available in the state of a composite entangled system than in the reduced states of its components. ${ }^{12}$ We can decompose the density operator associated with the singlet state, into

${ }^{10}$ Let us lay this objection aside and assume that it makes sense to read physical properties off nonphysical syntaxt, we still need a story as to why 'opposite spin' is selected and not 'definite and opposite spin?' Surely it is true of both $\uparrow \downarrow$ and $\downarrow \uparrow$ that the particles have definite and opposite spin, and thus should we not also say of the singlet state that its particles have definite and opposite spin? Yet this is false: the particles described by the singlet state do not have definite spin and may or may not have opposite spin (this is the contentious claim we are exploring.) This example highlights the danger inherent in inferring state properties from non-physical states.

${ }^{11}$ It might be the case that $Z_{-2}$ ought to be associated with 'opposite spin;' however, given our current understanding of quantum mechanics, we are not warranted in making this association. In order to be warranted, we require a more robust EE-link than the one we currently have.

${ }^{12}$ Hughes (1992, 150-151, 250). 
mixed density operators on its subsystems. Since the decomposition leads to a mixture it does not uniquely recompose into the composite singlet state. ${ }^{13}$ In other words, the singlet state as a whole is more tightly specified than the component systems. ${ }^{14}$ We can of course use the reduced density matrices to describe the subsystems but we cannot use them to describe the state as a whole. In order to describe the state as a whole, we need more information than can be obtained from the descriptions of the component subsystems. Thus, if we interpret an observable or relation defined on the full composite state as being about the component systems we are assuming that the operator does not have access to the full information of the state. ${ }^{15}$

In response, one could stipulate that the singlet state bears the relation 'opposite spin' and does so as a fundamental fact of the full state without reference to the spin of any particle. Under this interpretation, the relation is emergent and does not rest on the properties of the parts for its sense. We know that there are emergent properties of the system, so why should some of these not be weakly discerning relations? Of course, the possibility of their existence is not enough; in order to defend the PII, we require a demonstration that there is such a weakly-discerning-emergent-relation which holds of the singlet state. To be clear, this argument would need to demonstrate from only the information of the state ' $\Psi$ ', that some relation holds between its distinct particles and not between a particle and itself, and that this relation emerges from and is not directly produced by the single particle properties. Furthermore, even if Muller and Saunders were successful in arguing for such a relation, what would it have to do with their set of observables (10)? While this line

\footnotetext{
${ }^{13}$ Nielsen and Chuang $(2000,106)$.

${ }^{14}$ Hughes $(1992,151,250)$.

${ }^{15}$ One could get around this worry by showing that the missing information in going from the full state to the reduced states is just the correlation information captured by 'opposite spin.' One would argue that by predicating 'opposite spin' of the singlet state one captures both the information of the individual reduced states as well as the missing information by describing the correlations between the reduced states.
} 
of reasoning is an option, it is not metaphysically innocuous and for this reason alone Muller and Saunders will wish to avoid it. ${ }^{16}$

In summary, I have provided three challenges to predicating 'opposite spin' to states like the singlet state. The first challenge stems from Muller and Saunders' emphasis that their formal relation $Z_{-2}$ should be identified with a physical relation. I have argued that there is nothing in the formal structure of $Z_{-2}$ for thinking that it represents any physical property of the state, nor that it is a physical relation, nor that the physical relation is suitably structured to weakly discern. Secondly, I have noted that there is nothing in the singlet state itself to warrant thinking that the particles contained therein have opposite spin. Finally, I have argued that it is a mistake to predicate of an entangled state in terms of the information of its component systems: there is, in general, a loss of information. Thus, there are good reasons to reject Muller and Saunders' predication of 'opposite spin' to the relation $Z_{-2}$. Without a dyadic property of the singlet state, one cannot weakly discern the particles which compose it and Muller and Saunders' defense of the PII through weak discernibility, fails.

\footnotetext{
${ }^{16}$ Muller and Saunders' project is to defend the PII from quantum mechanics without recourse to dubious metaphysical assumptions (499).
} 


\section{References}

[1] Steven French and Michael Redhead. Quantum physics and the identity of indiscernibles. The British Journal for the Philosophy of Science, 39(2):233-246, 1988.

[2] Nick Huggett and Joshua Norton. Weak discernibility for quanta, the right way. British Journal for the Philosophy of Science, page axs038, 2013.

[3] R.I.G. Hughes. The Structure and Interpretation of Quantum Mechanics. Harvard University Press, 1992.

[4] Gottfried Leibniz. Discourse on metaphysics. (XV), 1686.

[5] N. David Mermin. What is quantum mechanics trying to tell us? American Journal of Physics, 66:753-767, 1998.

[6] M. Mugnai. Leibniz' Theory of Relations. Studia Leibnitiana: Supplementa. Franz Steiner, 1992.

[7] F. A. Muller and Simon Saunders. Discerning fermions. The British Journal for the Philosophy of Science, 59(3):pp. 499-548, 2008.

[8] M.A. Nielsen and I.L. Chuang. Quantum Computation and Quantum Information. Cambridge Series on Information and the Natural Sciences. Cambridge University Press, 2000.

[9] Willard V Quine. Grades of discriminability. The Journal of Philosophy, pages 113-116, 1976.

[10] Simon Saunders. Physics and leibniz's principles. In Symmetries in Physics: Philosophical Reflections, pages 289-307. Cambridge University Press, 2003a. 